\title{
Thyroid-SPOT for mobile devices: personalised thyroid treatment management app
}

\author{
Jia-Zhi Sim¹, Yu Zang ${ }^{1}$, Phi-Vu Nguyen ${ }^{1}$, Melvin Khee-Shing Leow ${ }^{3,4,5,6,7^{*}}$ and Samuel Ken-En Gan ${ }^{1,2^{*}}$
}

\begin{abstract}
Thyroid-SPOT is a mobile application that allows users to be able to analyse thyroid function tests laboratory reports by computing the homeostatic euthyroid set point based on regressing thyroid function data according to a patented algorithm to define the parameters that maximize the goodness-of-fit to a negative exponential model for targetcentric individualized treatment. The app facilitates analysis and optimization of treatment plans, while also allowing both patients and doctors to stay up to date with a function that allows ".txt" files to be imported and exported to and from Dropbox. The app improves treatment monitoring, allowing clinical analysis to be on-the go, thus boosting healthcare.
\end{abstract}

\section{Introduction}

Mobile apps are becoming increasingly popular in scientific research with great potential also for clinical uses (Gan SKE et al., 2015). There has already been apps developed that can help in clinical research (e.g. PsychVey, see Nguyen PV et al., 2015) as well as other that aid in diagnosis such as Peek Retina (2016) (https://www.indiegogo.com/projects/peek-retina-help-fight-avoidable-blindness\#/). In this fast-forwarded day and age, going to the doctor's clinic every week may not be a luxury many have: therefore, apps that allow doctors and patients to communicate clinically relevant information quickly and easily with each other are greatly desired. Thyroid-SPOT is one such app that links endocrinologists and patients together in managing their prescriptions, looking at thyroid hormone control issues. The fundamental law governing thyroid hormone physiology is a negative exponential function that connects "dose-response" feedback loop between free thyroxine (FT4) and thyroid-stimulating hormone (TSH) (Leow MK et al., 2007; Goede SL et al., 2014; Leow MKS et al., 2014). This model along with the set point equations derived using the maximum curvature theory form the background basis of a patented algorithm (Leow MKS

\footnotetext{
*Correspondence: melvin_leow@sics.a-star.edu.sg; samuelg@bii.a-star.edu.sg ${ }^{3}$ Singapore Institute for Clinical Sciences, Agency for Science, Technology, and Research (A*STAR), Singapore 117599, Singapore

${ }^{1}$ Bioinformatics Institute, Agency for Science, Technology, and Research (A*STAR), Singapore 138671, Singapore

Full list of author information is available at the end of the article
}

et al., 2013) that is programmed as the calculating engine of the Thyroid-SPOT software. A non-linear regression method of finding the exponential curve of best fit to the FT4/TSH coordinates is employed, from which the point of maximum curvature is determined according to the principles of differential calculus. These paired Android and $\mathrm{iOS}$ apps aid both groups in evaluating treatment with respect to individualizing doses that optimize thyroid function to a computed euthyroid set point unique to the individual. Details such as dates and times of doses in relation to thyroid function tests measurements can be stored for set point computation which facilitates accurate prescriptions. Coupled with the feature of importing and exploring ".txt" files to and from Dropbox (or "the cloud"), the app facilitates the viewing of profiles both on- and offline. This allows clinicians to boost their productivity and convenience by analysing and working "on-the-go". This in turn facilitates patient care as endocrinologists can now quickly know the euthyroid set point of any patient to aid in diagnosis and drug dosage prescription even while travelling between and within clinics and hospitals without spatial restriction to their desktops.

\section{Materials and methods}

Thyroid-SPOT App for Android was developed using 4.4 of the Eclipse Integrated Development Environment, Luna (https://eclipse.org/). 
Thyroid-SPOT App for iOS was developed using version 6.3.2 of the Xcode Integrated Development Environment, utilizing open source libraries: Core Plot 1.4 for the plotting and zooming of thyroid-spot graphs; DownPicker (https://github.com/Darkseal/DownPicker) for creating drop down lists.

\section{Create patient profile/ treatment entry}

Thyroid-SPOT Patient App offers patients a simple and user-friendly interface for the entry of demographic and clinical data with associated dates (see Figs. 1 and 2) including the treatment regime and the serum levels of free thyroxine (FT4) and thyroid stimulating hormone (TSH). Multiple data entries can be stored for the logging of treatments weeks in advance to aid in scheduling. Users can also import previous treatment details from Dropbox using a ".txt" file. This easily allows users to constantly stay updated with their treatments and thyroid function test results utilizing secure emails or data transfer methods without the need for physical records. Entered treatment details are displayed in a table showing the date and time of the treatments and corresponding serum FT4 and TSH values associated with those doses. Since different countries may use different units of measurement, there is an option for users to change the units of serum FT4 from pmol/L to ng/dL and vice versa based on a known conversion factor.

\section{Graph analysis (patient)}

The "View Entries" button reveals a line of best fit graph which plots the FT4 value on the X-axis and the TSH value on the Y-axis. Data points that follow fit in the line of best fit are marked in green with outliers marked in red. The graph can be enlarged or shrunk for in-depth visualization of specific data points. The data from the graph can then be exported to Dropbox for sharing by use of the smartphone communication avenues (e.g. WIFI, Bluetooth, NFC, internet).

\section{Patient selection (doctor version)}

The doctor version allows the importing of patient details saved as ".txt" files in the patient version. When a patient's data is accessed, the same table shown in the Patient app appears on the doctor's screen (see Fig. 3). However, the doctor version allows editing of the data points.

\section{Graph analysis (doctor)}

Clicking on the "View" button from the Patient Selection page will bring the user to a graph which is identical to the one shown in the patient app; however, the doctor version of the app also displays the patient's treatment and diagnosis (see Fig. 4). The calculated homeostatic euthyroid set point of the hypothalamus-pituitary-thyroid axis is a

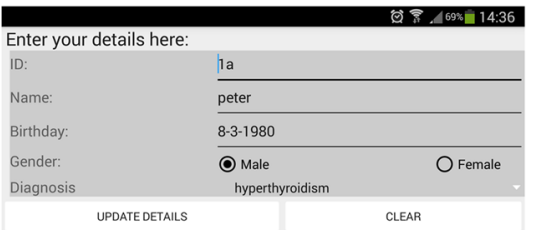

b Enter treatment details here:

Time: $\quad$ Enter Time here

FT4: $\quad$ Enter FT4 here

TSH: $\quad$ Enter TSH here

Drug: Please select:

Dose: $\quad$ Enter Dose here

\begin{tabular}{|c|c|c|c|}
\hline CREATE & VIEW & & EDIT \\
ENTRY & ENTRIES & IMPORT & PROFILE \\
\hline
\end{tabular}

Date Time FT4 TSH DELETE ENTRY

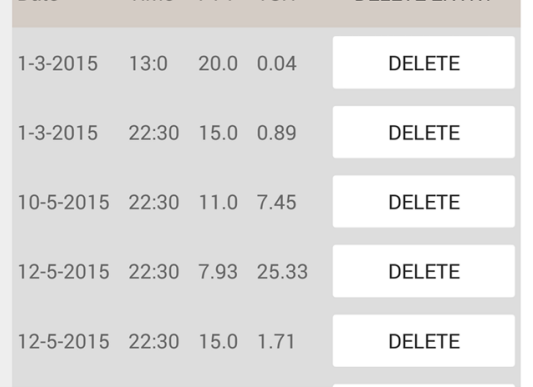

Cnter your details here:

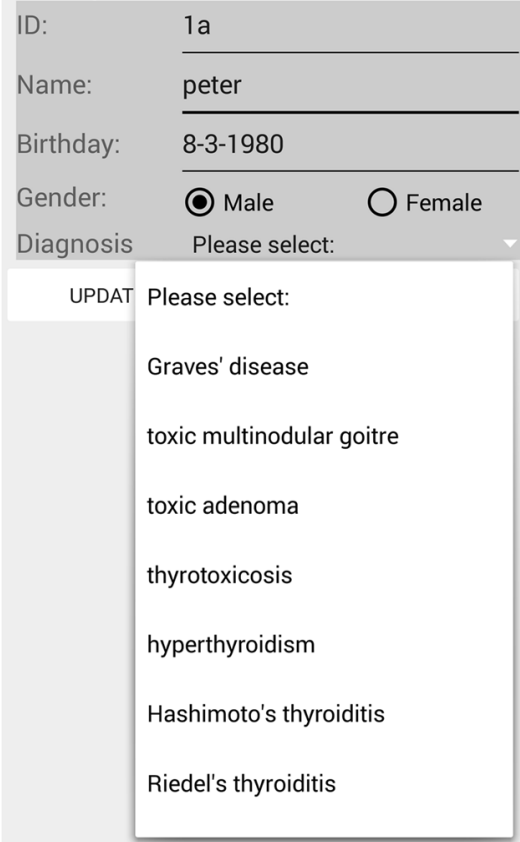

Fig. 1 Thyroid-SPOT Patient App's profile editing page and clinical data entry page. a Patient info entry page. b Patient test data entry page. c Disease drop down menu 
a

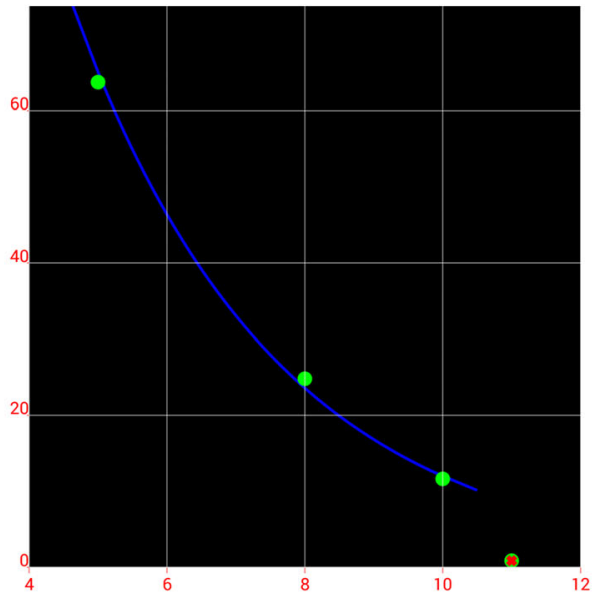

Best fit valid curve .Valid data .Outliers

Please contact and consult your clinic / clinician to decipher or export your results.

EXPORT DATABASE

b

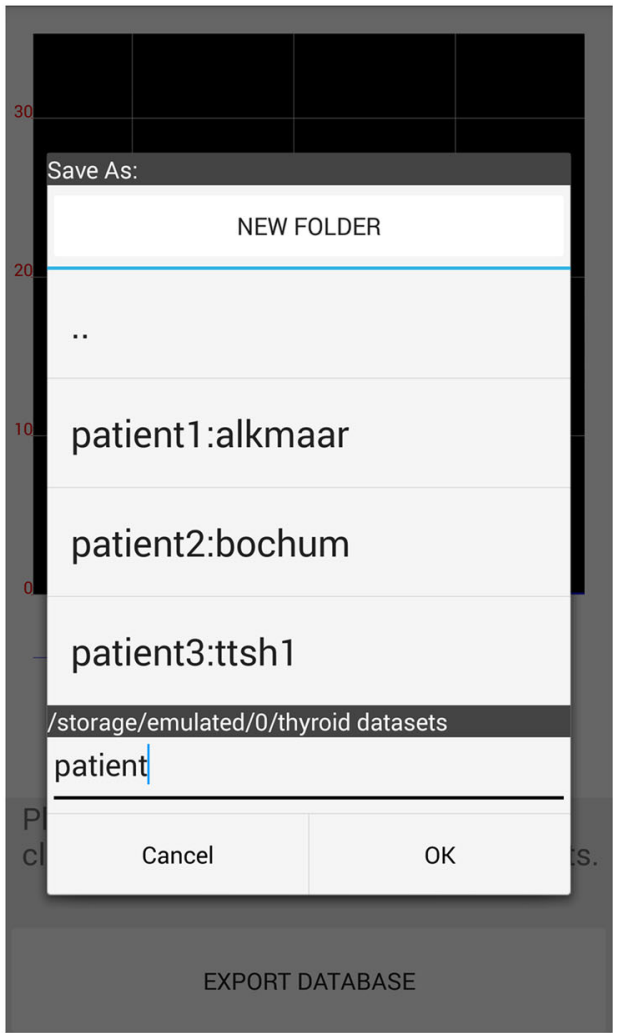

Fig. 2 Thyroid-SPOT Patient App's graph analysis page. a The calculated curve. To be interpreted and shown to the attending clinician. $\mathbf{b}$ Exporting of patient data a

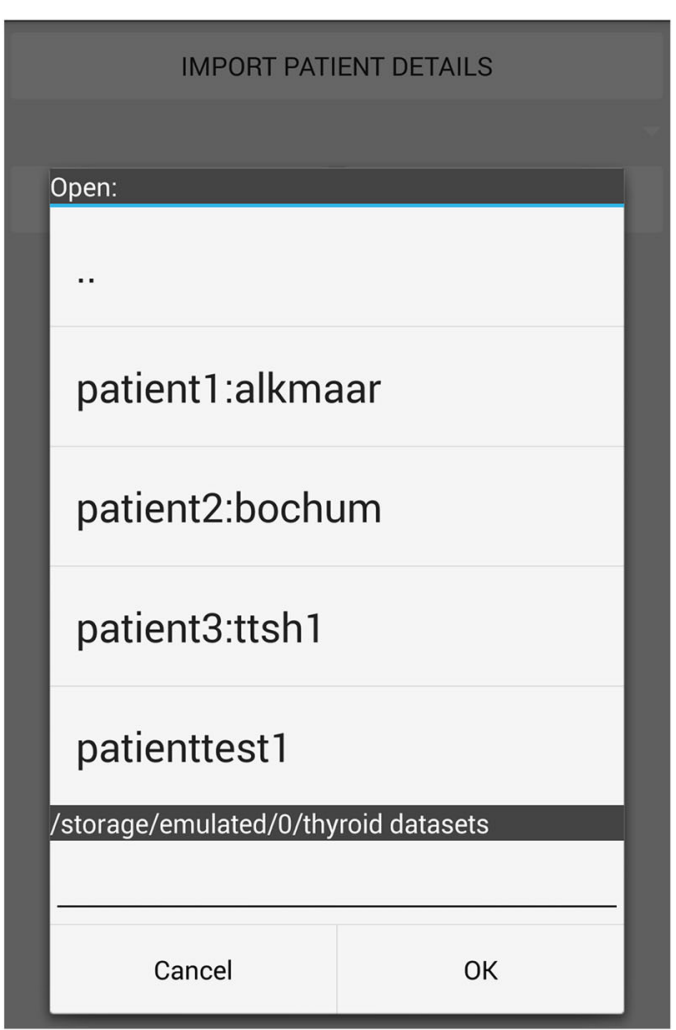

b

\begin{tabular}{|c|c|c|c|c|}
\hline \multicolumn{5}{|c|}{ IMPORT PATIENT DETAILS } \\
\hline \multicolumn{5}{|c|}{ s1234567c } \\
\hline & VIEW & & & DELETE \\
\hline Date & Time & FT4 & TSH & DELETE ENTRY \\
\hline $4-5-2015$ & $10: 54$ & 20.0 & 0.04 & DELETE \\
\hline $4-5-2015$ & $10: 54$ & 15.0 & 0.89 & DELETE \\
\hline $4-5-2015$ & $10: 54$ & 13.0 & 0.05 & DELETE \\
\hline $4-5-2015$ & $10: 54$ & 7.93 & 25.33 & DELETE \\
\hline $4-5-2015$ & $10: 54$ & 15.0 & 1.71 & DELETE \\
\hline $4-5-2015$ & $10: 54$ & 11.0 & 7.45 & DELETE \\
\hline $4-5-2015$ & $10: 54$ & 23.0 & 0.05 & DELETE \\
\hline $4-5-2015$ & $10: 54$ & 17.0 & 1.0 & DELETE \\
\hline
\end{tabular}

Fig. 3 Thyroid-SPOT Doctor App's Patient Selection. a Importing of patient details. $\mathbf{b}$ The entry tables of the blood test results 


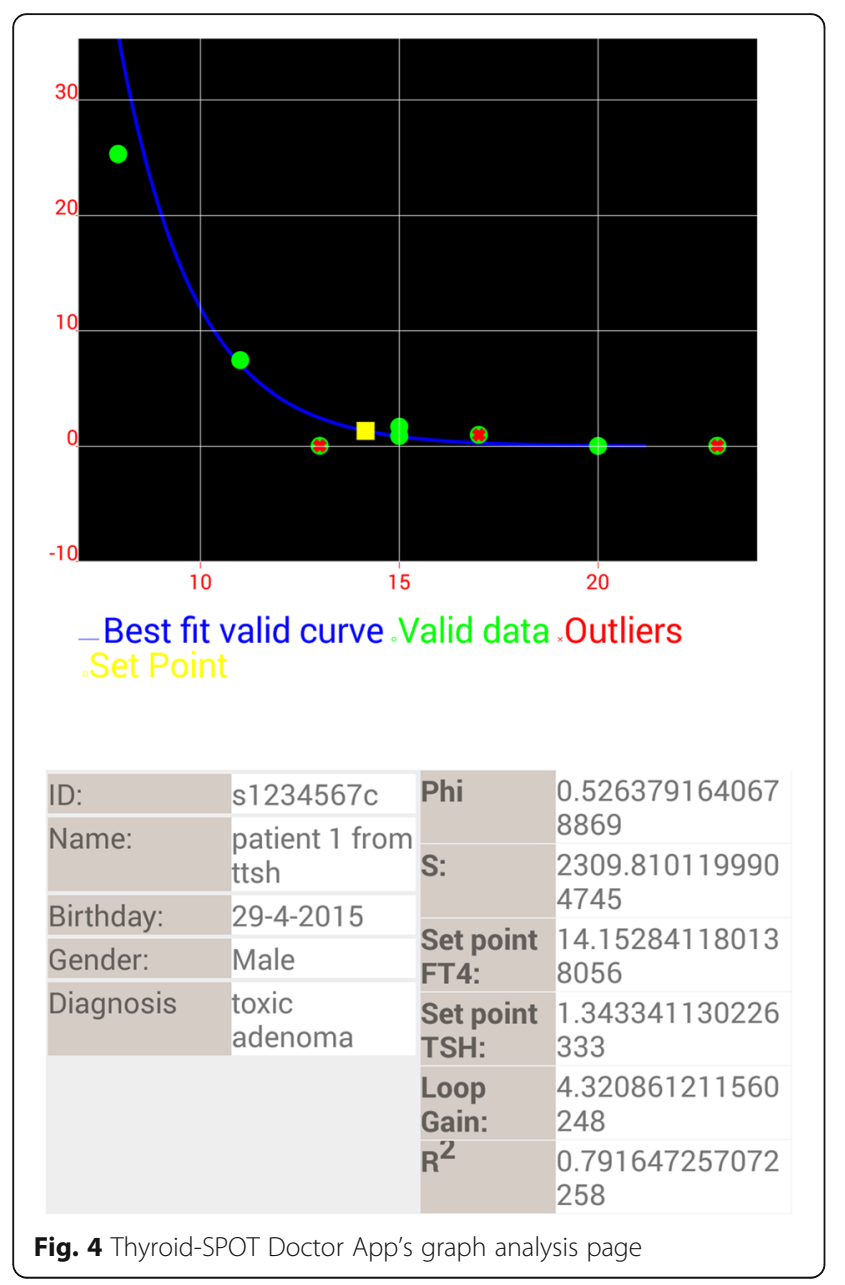

also displayed on the graph as a yellow square on the point of maximum curvature on the negative exponential curve. This is the most crucial aspect of the app as the set point forms the basis for dose titrations for personalized treatment targets for best thyroid health and metabolic outcomes.

\section{On-going clinical validations}

This mobile app is identical to the desktop counterpart in terms of its set point calculation function. The desktop version had been tested by one of the authors, who is a thyroidologist/endocrinologist and clinician scientist and it was useful for thyroid patients who continue to experience suboptimal quality of life. In this respect, the rapid computation of the euthyroid set point had anecdotally improved his patients' quality of life with diminished symptoms. In fact, the underlying patented and previously published Thyroid-SPOT algorithm was clinically validated in a number of clinical cases as published in two earlier papers (Goede SL et al., 2014; Leow MKS et al., 2014). This endocrinologist co-author is presently conducting a formal randomized double-blind placebo controlled study of the effectiveness of the ThyroidSPOT algorithm entitled EQUILEBRATE, a mnemonic which stands for "A Prospective Randomized, Doubleblind, Parallel Arm, Multi-centre Clinical Trial to Evaluate the Quality of Life and Euthyroid Balance using Conventional Thyroid Hormone Replacement versus Set Point Strategy". The patients recruited for EQUILEBRATE are mainly those who have primary hypothyroidism being replaced with L-thyroxine. Quality of life in this clinical trial is evaluated using a robust questionnaire (SF-36) as well as a thyroid-specific questionnaire called ThyPro (Watt $\mathrm{T}$ et al., 2010). In yet another study codenamed TRIBUTE which stands for "The Role of Thyroid Status in Regulating Brown Adipose Tissue Activity. White Adipose Tissue Partitioning and Resting Energy Expenditure", patients with hyperthyroidism from Graves' disease are being assessed for both residual thyroid symptoms and objective measures of fasting lipids, serum electrolytes, body weight and brown adipose tissue function as they undergo anti-thyroid drug therapy such that the patients will then be assessed on how close or far apart their thyroid function tests are from the computed set point and this will in turn be correlated with those objective measures as elaborated above. Finally, there are efforts ongoing to prove the accuracy of the set point prediction by comparing the computed set points of thyroidectomised patients on Lthyroxine replacement to the thyroid function tests prethyroidectomy at a time when these patients were healthy, well and euthyroid some years prior, in a retrospective study entitled PREDICT-IT which stands for "Profiling Retrospective Thyroid Function Data in Complete Thyroidectomy Patients to Investigate the HPT axis Set Point". As such, to facilitate the use of the algorithm and for convenience, mobile versions have been created and were found to be exceptionally handy on-the-go while attending to a phone specialist consultation or whenever immediate assessment if a given dose of thyroid medication might be either suboptimal or excessive for a given patient located in another hospital or clinic during 'on-call' duties.

\section{Limitations}

Some limitations to this app include the difficulty of computing the euthyroid set point with very high precision if the thyroid function tests of a patient were performed in different laboratories and at varying times of the day. This is due to the different thyroid function tests reports if the sample of blood was split into separate vials and simultaneously submitted to different assay platforms. In addition, the concentrations of these thyrotropic hormones can differ significantly when sampled at different times of the day due to a natural diurnal rhythm. 
Of relevance here is the possible misperception that the TSH range is "narrow" and that both L-thyroxine and antithyroid drugs come in fixed doses which limits the scope of the Thyroid-SPOT algorithm. The TSH range is in fact very wide with a 16 -fold difference between the lower and upper boundaries of the normal serum TSH range (0.34-5.60 mIU/L). Even though the doses of L-thyroxine may come in fixed doses (eg. $25 \mathrm{mcg}, 50 \mathrm{mcg}, 75 \mathrm{mcg}$, and $100 \mathrm{mcg}$ tablets), dose adjustments and fine-tuning are indeed possible. For instance, a patient who requires a dose of $62.5 \mathrm{mcg}$ daily may either be prescribed one and a half tablets of the $50 \mathrm{mcg}$ strength pill. Alternatively, because of the very long half-life of L-thyroxine ( $\sim 7$ days), it is possible to dose the patient with $50 \mathrm{mcg}$ daily alternately with $75 \mathrm{mcg}$ daily. Should the patient require other doses such as $57 \mathrm{mcg}$ daily, he/she can be prescribed 50 mcg daily for 5 days (weekdays) and 75 mcg daily for 2 days (weekends). Similarly, anti-thyroid drugs such as thiamazole only comes in strengths of $5 \mathrm{mg}$ and $10 \mathrm{mg}$. However, depending on the dose required, a patient may be prescribed $2.5 \mathrm{mg}$ (half a pill) to $40 \mathrm{mg}$ (4 pills) daily. A reasonable range of dose permutations and combinations are possible to allow for doses that fall outside the typical standard doses offered by the strengths of these thyroid pills. In this fashion, one can titrate the doses of these medications to attain serum FT4 and TSH concentrations that are as close as possible to their computed set points.

\section{Conclusion}

Thyroid-SPOT is a clinical-aid tool for both clinicians and patients. These paired apps help both groups keep au courant with treatment progress, aiding in analysis and off-line storage of data, all while allowing users to back up all the information to Dropbox. With the function to enter multiple treatments at one time, the app helps patients and doctors to stay organised, even when off-line.

\section{Funding}

This work is funded by Agency for Science, Technology, and Research

(A*STAR), Singapore

We thank Mr. Dillon Lim for his help in drafting these notes.

\section{Availability of data and materials}

The versions are freely available to verified clinicians by request at email to melvin_leow@sics.a-star.edu.sg. The user guide can be found at tinyurl.com/ ThyroidSPOT. For the purpose of the review, the IPA file for the iOS versions are placed: http://tinyurl.com/ThyroidSOTDrviOS and http://tinyurl.com/ThyroidSOT ptviOS. The Android Doctor version is available: http://tinyurl.com/ThyroidSOT DrvAnd. Contact: For more information, please email: bii-apdlab@bii.a-star.edu. sg; samuelg@bii.a-star.edu.sg; melvin_leow@sics.a-star.edu.sg; Supplementary information: More details on the app can be found at www.facebook.com/ APDLab; www.bii.a-star.edu.sg/research/trd/apd.php. The Thyroid-SPOT user guide is available from the "About Us" page of the apps.

\section{Authors' contributions}

SIM JZ made and designed the Android app. Zang Y made the iOS version of the app. Nguyen PV assisted in the implementation of the graph on both Android and iOS apps. Leow M provided the documentation on which the algorithm for the apps were made. Gan SKE oversaw and directed the design and making of the apps. The authors have read and approved of the manuscript.

\section{Competing interests}

The authors declare that they have no competing interests.

\section{Author details}

${ }^{1}$ Bioinformatics Institute, Agency for Science, Technology, and Research (A*STAR), Singapore 138671, Singapore. ${ }^{2}$ p53 Laboratory, Agency for Science, Technology, and Research (A*STAR), Singapore 138648, Singapore.

${ }^{3}$ Singapore Institute for Clinical Sciences, Agency for Science, Technology, and Research (A*STAR), Singapore 117599, Singapore. ${ }^{4}$ Department of Endocrinology, Division of Medicine, Tan Tock Seng Hospital, Singapore 308433, Singapore. ${ }^{5}$ Lee Kong Chian School of Medicine, Nanyang Technological University, Singapore 639798, Singapore. ${ }^{6}$ Yong Loo Lin School of Medicine, National University of Singapore, Singapore 119228, Singapore.

${ }^{7}$ Duke-NUS Medical School, Singapore 169857, Singapore.

Received: 18 January 2017 Accepted: 1 August 2017

Published online: 30 August 2017

\section{References}

Gan SKE. Editorial: scientific apps: design, considerations, and functions. Scientific Phone Apps and Mobile Devices. 2015;1(1):1. doi:10.1186/s41070-015-0001-2.

Goede SL, Leow MK, Smit JW, Dietrich JW. (2014) A novel minimal mathematical model of the hypothalamus-pituitary-thyroid axis validated for individualized clinical applications. Math Biosci. 2014 Mar;249:1-7. doi: 10.1016/j.mbs.2014.01.001.

Leow MK. A mathematical model of pituitary-thyroid interaction to provide an insight into the nature of the thyrotropin-thyroid hormone relationship. J Theor Biol. 2007;248(2):275-87.

Leow MK, Goede SL. (2014). The homeostatic set point of the hypothalamuspituitary-thyroid axis-maximum curvature theory for personalized euthyroid targets. Theor Biol Med Model. Aug 8;11:35. doi: 10.1186/1742-4682-11-35.

Leow MKS, Goede SL and Dietrich JW (2013) System and method for deriving parameters for homeostatic feedback control of the hypothalamic-pituitarythyroid axis of an individual, regular patent application no: PCT/SG2013/000515.

Nguyen PV, Lim JPH, Budianto IH, Gan SKE (2015). PsychVey: research survey app. Scientific phone apps and mobile devices, 1:3, doi:10.1186/s41070-015-0002-1.

Peek Retina: Help fight avoidable blindness" (2016). Available: https://www.indiegogo. com/projects/peek-retina-help-fight-avoidable-blindness\#/. Accessed 1 Mar 2016.

Watt T, Hegedüs L, Groenvold M, Bjorner JB, Rasmussen AK, Bonnema SJ, FeldtRasmussen U. Validity and reliability of the novel thyroid-specific quality of life questionnaire, ThyPRO. Eur J Endocrinol. 2010;162(1):161-7. doi:10.1530/ EJE-09-0521. Epub 2009 Oct 1.PMID: 19797502

\section{Submit your manuscript to a SpringerOpen ${ }^{\mathcal{O}}$ journal and benefit from:}

- Convenient online submission

Rigorous peer review

- Open access: articles freely available online

- High visibility within the field

- Retaining the copyright to your article

Submit your next manuscript at $>$ springeropen.com 